\title{
Psychosocial functioning, quality of life and clinical correlates of comorbid alcohol and drug dependence syndromes in people with schizophrenia across Europe \\ Findings from the European Schizophrenia Cohort (EuroSC)
}

\begin{abstract}
Little is known about the correlates of comorbid drug and alcohol dependence in people with schizophrenia outside the USA. We tested hypotheses that dependence on alcohol or drugs would be associated with more severe symptoms, and poorer psychosocial functioning and quality of life. The EuroSC Cohort study $(\mathrm{N}=1,204)$, based in France, Germany and the UK, used semi-structured clinical interviews for diagnoses, and standardized tools to assess correlates. We used mixed models to compare outcomes between past-year comorbid dependence on alcohol or drugs, controlling for covariates and modelling both subject and country level effects. Participants dependent on alcohol or drugs had fewer negative symptoms on PANSS than their non-dependent counterparts. However, those dependent on alcohol scored higher on PANSS general psychopathology than those who were not dependent, or dependent only on drugs. People suffering from schizophrenia dependent on drugs had poorer quality of life, more extrapyramidal side effects, and scored worse on GAF than those without dependence. People with alcohol dependence reported more reasons for noncompliance with medication, and poorer functioning on Global Assessment of Functioning (GAF), though not on Global Assessment of Relational Functioning (GARF). In people with schizophrenia, comorbid dependence on alcohol or drugs is associated with impaired clinical and psychosocial adjustment, and poorer quality of life.
\end{abstract}

Keywords: schizophrenia; alcohol dependence; drug dependence; comorbidity 


\section{Introduction}

A substantial proportion of people with schizophrenia misuse alcohol and other drugs (Green et al., 2005; Swartz et al., 2006a; Koskinen et al., 2009; Koskinen et al., 2010), though this varies between different settings and geographical areas (Carrà et al., 2012). However, this body of research has been troubled by methodological issues. Samples have often been based on clinical convenience rather than on epidemiological principles, involving small sample sizes, and recruiting from specific settings and locations (Carrà and Johnson, 2009). In addition, the assessment of substance use has often been inadequate, with relatively few studies making full diagnostic assessments (Drake et al., 1993). Moreover, correlates have been described in relation, variously, to point, period and life-time prevalence (Goldfinger et al., 1996), and dependence disorders involving alcohol and other drugs have frequently not been distinguished from DSM-IV abuse (ICD-10 harmful use), though the consequences of dependence may be much more severe than those of harmful use in people with schizophrenia (Olfson et al., 2002; Potvin et al., 2006a; Kerfoot et al., 2011; Carrà et al., 2015). Indeed, DSM 5 has combined DSM-IV substance abuse and dependence into a single disorder, measured on a mild to severe continuum of severity (Hasin et al., 2013; Bartoli et al., 2015). Though the bulk of the evidence is from the US, a significant body of research has now built up in Europe (i.e., Soyka et al., 2001; van Os et al., 2002; Weaver et al., 2003; Morrens et al., 2011). This has contributed to the development of specialized treatment programs for these complex clinical populations, whose models of comorbidity are not entirely clear (Carretta et al., 2015). The characteristics of clinical populations with both schizophrenia and substance use disorders are universally worse than their non-abusing counterparts (Margolese et al., 2004; Kerner, 2015), including clinical (Kerfoot et al., 2011; Jones et al., 2011), physical (Rosenberg et al., 2001) and legal (Carrà et al., 2015) outcomes. Nonetheless, it has been suggested that psychosocial functioning and quality of life (QoL) might actually be better in substance-abusing people with schizophrenia than in those who are abstinent, given that they need the skills to engage with drugdealers in order to secure supplies (Salyers and Mueser, 2001; Swartz et al., 2006b). However, this might only be true in those people with schizophrenia who misuse drugs and alcohol rather than 
those with actual dependence. The issue has been clouded, both by the question of cross-cultural applicability (Heinrichs et al., 1984), and by problems in content validity (Schooler et al., 1979). Finally, since the correlates of dependence on alcohol in the general population differ from those of dependence on other drugs (Rehm et al., 2006), this may also be the case in people with schizophrenia.

More precise knowledge of the correlates of comorbid substance misuse should increase understanding of the impact of local social circumstances on these associations, and the identification of clinical subpopulations with residual competencies and skills, suitable for targeting with specialist treatment programmes (Carrà et al., 2006).

The European Schizophrenia Cohort (EuroSC) survey provides an opportunity to do this, as it was specifically set up to compare the attributes and correlates of schizophrenia in three European countries, France, Germany and the UK (Bebbington et al., 2005). Using a large and representative sample of people with schizophrenia in community mental health care, it showed lifetime rates of $35 \%$ for comorbid dependence on any substance in the UK, but considerably lower values in Germany (21\%) and in France (19\%). These differences between countries persisted after controlling for individual clinical and demographic characteristics (Carrà et al., 2012).

Our aim in the current study was to use the baseline EuroSC data to investigate cross-nationally the socio-demographic, clinical, social functioning and quality of life (QoL) correlates of dual diagnosis in people with schizophrenia. We examined dependence on alcohol and on other drugs separately. We hypothesised that, after adjusting for country of residence, people with schizophrenia and comorbid dependence would have significantly worse clinical symptoms, psychosocial functioning, and quality of life. As a secondary hypothesis, we predicted that drugs would be associated with fewer impairments in psychosocial functioning and QoL than dependence on alcohol.

\section{Methods}




\subsection{Sample}

The European Schizophrenia Cohort (EuroSC) survey was a naturalistic follow-up of a cohort of people aged 18 to 64, suffering from schizophrenia, and in contact with secondary psychiatric services, i.e., community outpatient services according to national organizational standards, in nine community mental health catchment areas in France, Germany, and the UK (Bebbington et al., 2005). The current analysis is based on cross-sectional data from the first stage interview. Local ethical approval for the study was obtained in each country. The settings, sampling strategies and inclusion/exclusion criteria are fully described elsewhere (Bebbington et al., 2005). In brief, in France, people were recruited from three centres located in a city or in medium-size towns from northern (Lille), central (Lyon), and southern France (Marseille). In Germany four catchment areas were identified for the study: two in the former East Germany (Leipzig and the nearby Altenburg area) and two in the former West (Hemer and the County of Heilbronn). The British study centres were Islington, an inner-city area of London, and the reasonably affluent area of Leicestershire minus the city of Leicester. Random sampling from lists of service users was adopted in all the French centres and in London, while an exhaustive inclusion strategy was used for the German centres and Leicestershire. Eligible patients were aged 18-64 years at the time of enrolment in the study, had a diagnosis of schizophrenia according to DSM-IV criteria, and had given signed informed consent. People who had been hospitalised for the past 12 months, or were currently intoxicated, roofless or planning to leave the area, making follow-up assessment impracticable, were excluded.

\subsection{Procedure}

Individuals from the final list of participants were contacted consecutively by trained research assistants, seeking their informed consent, and fully reassuring them about their privacy protection also with the help of local clinicians if needed. If they agreed, they were interviewed at home or in a clinical facility over approximately three hours. Assessments sometimes required more than one session in order to avoid impairment of level of attention and willingness to collaborate. The study was observational, as no intervention was made either by, or at the behest of, the research team. 


\subsection{Instruments}

An extensive battery of instruments was used to collect information during face to face interviews. Only those relevant to this paper are presented here. The Diagnostic interviews: SCAN - Schedules for Clinical Assessment in Neuropsychiatry - version 1.0 (WHO, 1992) was used to evaluate the 4week period before interview and the most significant period of earlier psychopathology. In the UK and Germany, SCAN was used with its component algorithm to establish diagnoses of schizophrenia. In the French centres, only the SCAN sections on alcohol and drug use were used, and the Structured Clinical Interview for DSM-IV (Spitzer et al., 1992) was used to identify schizophrenia. In all three countries, SCAN 1.0 algorithms were used to derive diagnoses of comorbid dependence on alcohol and on psychoactive substances other than alcohol. Information on current symptom profile was collected through the 30 item Positive and Negative Syndrome Scale (PANSS) (Norman et al., 1996), with positive, negative and general psychopathology symptoms sub-scores. The Quality of Life Interview (QoLI - Lehman, 1983) provided a global measure of life satisfaction, with a higher score indicating a better overall quality of life. The Calgary Depression Scale for Schizophrenia (CDSS) was used to measure depression (Addington et al., 1990, 1992). The Rating of Medication Influences (ROMI) Scale (Weiden et al., 1994) evaluated adherence to medication, with outputs scoring total "reasons for compliance" and "reasons for non-compliance": higher scores signified, respectively, a greater willingness or reluctance, to take medication. The Clinical Global Impression (CGI) gives a single overall rating of the degree of mental illness on a 7-point scale (Busner and Targum, 2007). The Global Assessment of Functioning (GAF) provided an overall evaluation of functioning for people with psychiatric disorders, intentionally combining mental status and social function (APA, 1994). We also used the Global Assessment of Relational Functioning (GARF), which quantifies the relational context within which patients live and problems occur (Dausch et al., 1996), rating the degree to which the family (broadly defined) meets the affective and instrumental needs of its members in the areas of joint problem solving, organisation and emotional climate. Finally, the Extrapyramidal Rating Scale of Simpson and Angus (1970) was used to assess neuroleptic-induced Parkinsonism. 


\subsection{Definitions of dual diagnosis}

We chose to focus on dependence rather than on harmful use of substances, because the latter is a residual diagnosis that cannot be made in people who meet criteria for dependence. Moreover, while the diagnosis of dependence, as defined in DSM-IV and ICD-10, has consistently been shown to be reliable and valid, reliability is far weaker for harmful use (abuse in DSM-IV) (Hasin et al., 2006).

Our definitions of dual diagnosis were based on ICD-10 diagnoses of past year comorbid dependence due to the use of (1) alcohol, or (2) any psychoactive substance other than alcohol (F10-F16, F18-F19). We did not collect data about disorders due to the use of tobacco.

Furthermore, because of high frequency of polysubstance dependence, dependence on specific substances of abuse could not be assessed. For brevity, in the text, tables and figures, we refer to alcohol dependence and drug dependence. In order to analyse mutually exclusive subgroups, people with both alcohol and drug dependence were assigned to the category of the most severe syndrome.

\subsection{Analysis}

Univariate analyses were carried out testing whether past year dependence syndromes on alcohol and on other drugs were associated with baseline characteristics by country. Univariate comparisons for categorical data were made between groups using Pearson's chi-square test, and Student $\mathrm{t}$ and Mann-Whitney tests for continuous variables. In order to control for error effects, the Bonferroni multiple testing correction was used.

Multivariate analyses were then used to test the association between different correlates and the two different, past year dependence syndromes, controlling for country. Multinomial logistic regression was used to identify a parsimonious subgroup of predictors among sociodemographic and clinical variables characterizing mutually exclusive groups of dependent people (i.e., individuals dependent on alcohol and on other drugs) in comparison with people without dependence. In order to identify the relevant correlates of the two dependence syndromes we followed a structured approach. For each psychosocial and clinical correlate, we fitted a multilevel mixed effects linear regression, modelling subject-level and country-level effects, and including identified predictors as covariates, 
in order to compare outcomes between the three groups (past year no dependence, dependence on alcohol, dependence on other drugs). Least square means for each predictor across groups were estimated, with p-values for joint tests. In addition, comparisons were conducted between pairs of groups, providing Bonferroni multiple testing corrections. Level of significance was set at 5\%. Analyses were performed using the Stata statistical software package (version 13.0; StataCorp, College Station, Texas).

\section{Results}

In total, 1,204 people with schizophrenia participated in the study, 284 in France, 302 in the UK, and 618 in Germany. In general, participants had suffered from schizophrenia for a considerable period (13.5-16.6 years). There were minor but significant differences in the sociodemographic and clinical characteristics: nearly all varied significantly according to centre of residence. Further details are described elsewhere (Bebbington et al., 2005).

\subsection{Univariate group comparisons}

Paired comparisons showed significant differences between groups on 13 of 22 measures (Table 1). Generally, dependent participants were younger (significantly so for drug dependence), male, and single. Dependent participants living in France and Germany had a shorter duration of illness. Participants dependent on either alcohol or drugs had PANSS general psychopathology and positive subscale scores higher than those who were not dependent. Dependent individuals also had higher CDSS scores, poorer quality of life (QoL) scores, and worse scores on CGI, GAF, and GARF. There were no differences between groups in medication patterns and extrapyramidal rating scores. Multinomial logistic regression confirmed these independent differences between groups on the following measures: gender, family situation, living condition, age, ever worked, years of education, age of illness onset, length of illness, PANSS positive and negative, overall QoL, CGI, and GAF scores (all $\mathrm{p}<0.05$; data available upon request). 


\subsection{Comparison of correlates}

Controlling for identified covariates, and incorporating both subject and country level effects, we used mixed models to compare outcomes between the drug dependent, alcohol dependent and nondependent groups. There were significant differences on the PANSS negative and general psychopathology subscales, and in quality of life (QoL), non-compliance with medication (ROMI), extrapyramidal side effects (EPS), and functioning on both the GAF and the GARF (Table 2). However, relative to the non-dependent group, people suffering from schizophrenia who were dependent on either alcohol or drugs had fewer symptoms on the PANSS negative subscale. Participants dependent on alcohol scored higher on the general psychopathology subscale than their drug-dependent and non-dependent counterparts. Compared to non-dependent participants, those dependent on drugs reported poorer quality of life, more extrapyramidal side effects, and poorer functioning on the GAF; people dependent on alcohol gave more reasons for non-compliance with medication, and reported poorer GAF functioning. However, there were no group differences on the GARF, which quantifies relational functioning in terms of problem solving, organisation and emotional climate.

\section{Insert Table 2 here}

\section{Discussion}

We analysed a large international sample of people with schizophrenia, involving three European countries. ICD-10 and DSM-IV equivalent research diagnoses for schizophrenia and for comorbid dependence were based on formal diagnostic interviews. Taking into account country of residence, we were thus able to study psychosocial functioning, quality of life and the clinical correlates associated with concurrent past-year dependence on alcohol and other, illegal, substances.

Our results show that people suffering from schizophrenia with comorbid dependence were more likely to be male, younger, and single, with shorter illnesses, more severe clinical symptoms, and poorer QoL and psychosocial functioning. People dependent on alcohol or on drugs had fewer 
symptoms on the PANSS negative subscale. On the general psychopathology subscale, participants dependent on alcohol scored higher than those dependent on drugs or with no dependence. Notably, there was no excess of PANSS positive symptoms in people suffering from schizophrenia with comorbid alcohol or drug dependence. Findings of fewer negative symptoms in comorbid substance users tally with the bulk of the literature (e.g., Swartz et al., 2006b; Kerfoot et al., 2011), which suggests a correspondence between the most severe dependence syndromes and attempts to alleviate dysphoria (e.g., Potvin et al., 2006a; Talamo et al., 2006). Nevertheless, fewer negative symptoms may reflect better premorbid functioning, in that drugs-using patients may have superior social skills in order to be able to acquire and sustain a drug habit (Salyers and Mueser, 2001). A rigorous assessment of comorbid dependence on alcohol or other drugs could thus help clinicians in order to anticipate typical clinical features which might orientate the choice of active components in treatment programs. These may include care in the community, assertive engagement, high intensity, small caseload, continuous responsibility and availability, consistent multidisciplinary team, a team approach, and cooperation with the patient's support network (De Witte et al., 2014). In particular different levels of positive rather than negative psychotic symptoms on PANSS would also demand optimization of antipsychotic medication (Lingford-Hughes et al., 2012). However, only people suffering from schizophrenia who had comorbid alcohol dependence showed a significant impairment on the general psychopathology subscale, in which the items address general functional areas (e.g. cognitive, social, affective, and communicative). This is in line with findings linking alcohol dependence attributes such as craving with global psychiatric symptom severity (Batki et al., 2008). Along with the greater degree of EPS after controlling for medication status (Potvin et al., 2006b), it may also explain the associated medication non-compliance we found, consistent with previous research (Buckley, 2006).

In contrast to many previous US samples (e.g., Swarz et al., 2006b; Talamo et al., 2006; Kerfoot et al., 2011), but consistent with some European studies (e.g., Dervaux et al., 2002), we did not find significantly higher PANSS-positive scores in our sample of schizophrenic subjects with comorbid dependence. However, more recent investigations from the USA, using latent class analysis based 
on the CATIE study (Tsai and Rosenheck, 2013) and the Molecular Genetics of Schizophrenia (MGS1) Collaboration study (Kerner, 2015) have also reported that comorbid substance use was unrelated to any specific pattern of positive symptoms. The findings in our study and in others might result from the relatively high level of positive symptoms consequent on the selection criteria, and may represent a floor effect. Our results do not entirely corroborate prior research in people with schizophrenia misusing substances indicating more social contacts and better socialleisure functioning (possibly proxy measures of QoLI), albeit with coexistent more interpersonal and family problems (Salyers and Mueser, 2001) and higher overall psychosocial functioning (Swartz et al., 2006b). Indeed, regardless of country of residence, participants dependent on drugs or on alcohol had significantly lower QoL overall scores and poorer functioning on the GAF relative to their non-dependent counterparts. In contrast, there were no differences in relational functioning, as measured by the GARF. Thus our secondary hypothesis that people with schizophrenia and comorbid dependence (in particular those dependent on drugs) would have fewer impairments in psychosocial functioning and QoL was clearly refuted.

It is possible that the degree of programming, organization, and social performance required for the acquisition of illicit drugs (Swartz et al., 2006b), may apply only to drug misuse, and not to drug dependence as analysed in our study.

\subsection{Limitations}

We acknowledge several limitations. First, we had no access to information on people who declined to participate in the study. While attempts were made to guarantee comparable recruitment procedures across the centres of the study, variations in the inpatient services available for addiction cannot be excluded. This could be controlled to an extent by controlling for country of study, but more specific differences in the availability of clinical resources might be included as potential confounding factors in future research. The procedure for identifying schizophrenia was based on the use of different standardised instruments (SCID and SCAN) that may identify different cases, although these are likely to be similar in their correlates (the identification of substance dependence was uniform across 
the three countries). The role of possible confounders has been addressed at the stage both of design (random sampling and exhaustive inclusion procedures) and of analysis (adjusting for a selected set of variables at subject-level and country-level). We did not cover special populations (e.g., prisoners, homeless, and those in residential long-term programmes) who may perhaps have more severe forms of substance disorders (Mojtabai, 2005), and we could not collect information about treatment in place for the dependence syndrome. In addition, because of idiosyncratic characteristics in the design and financing of European health services, our results may not generalize to other settings. Finally, limitations regarding temporal relationship and causality, inherent to cross-sectional design, should obviously be considered, and findings might not be the same in people out of contact with services. Future research should be planned in order to fill these gaps in this field of the scientific literature.

\subsection{Conclusions}

We have previously reported that in people with schizophrenia dependence on alcohol and other drugs is less frequent in Europe than in the USA (Carrà et al., 2012), though differential access to mental health systems might in part explain differences in prevalence rates (Carrà \& Johnson, 2009). This study extends this research by suggesting that, at least across Europe, people with schizophrenia who are dependent on alcohol or drugs are more disabled than those who are not dependent. 


\section{Acknowledgments}

Our thanks to all the patients and staff that helped with the study and to the Camden and Islington Mental Health and NHS Foundation Trust and the Leicestershire Partnership NHS Trust R\&D

Programme and to those who collected the data and otherwise contributed to the main study.

\section{Financial support}

This study was funded by grants from Lundbeck A/S and from the German Federal Ministry of Education and Research.

\section{Conflict of interest}

Giuseppe Carrà, Sonia Johnson, Cristina Crocamo, and Traolach Brugha have not received any financial support by pharmaceutical companies in recent years. Paul Bebbington and Matthias C. Angermeyer are in retirement and have not received any financial support by pharmaceutical companies in recent years. Jean- Michel Azorin has received unrestricted research grants from $\mathrm{H}$. Lundbeck A/S. Mondher Toumi was an employee of H. Lundbeck A/S. 


\section{REFERENCES}

- Addington, D., Addington, J., Maticka-Tyndale, E., Joyce, J., 1992. Reliability and validity of a depression rating scale for schizophrenia. Schizophr Res 6, 201-208.

- Addington, D., Addington, J., Schissel, B., 1990. A depression rating scale for schizophrenics. Schizophr Res 3, 247-251.

- American Psychiatric Association (APA), 1994. Diagnostic and statistical manual of mental disorders, 4th edn, revised. American Psychiatric Association, Washington, DC.

- Bartoli, F., Carrà, G., Crocamo, C., Clerici, C., 2015. From DSM-IV to DSM-5 alcohol use disorder: An overview of epidemiological data. Addict Behav 41, 46-50.

- Batki, S.L., Leontieva, L., Dimmock, J.A., Ploutz-Snyder, R., 2008. Negative Symptoms are Associated with Less Alcohol Use, Craving, and "High" in Alcohol Dependent Patients with Schizophrenia. Schizophr Res 105, 201-207.

- Bebbington, P.E., Angermeyer, M., Azorin, J.M., Brugha, T., Kilian, R., Johnson, S., Toumi, M., Kornfeld, A.; EuroSC Research Group, 2005. The European Schizophrenia Cohort (EuroSC): a naturalistic prognostic and economic study. Soc Psychiatry Psychiatr Epidemiol 40, 707-717.

- Buckley, P.F., 2006. Prevalence and consequences of the dual diagnosis of substance abuse and severe mental illness. J Clin Psychiatry 67 Suppl 7, 5-9.

- Busner, J., Targum, S.D, 2007. The Clinical Global Impressions Scale: Applying a Research Tool in Clinical Practice. Psychiatry (Edgmont) 4, 28-37.

- Carrà, G., Crocamo, C., Borrelli, P., Popa, I., Ornaghi, A., Montomoli, C., Clerici, M., 2015. Correlates of dependence and treatment for substance use among people with comorbid severe mental and substance use disorders. Compr Psychiatry 58, 152-159.

- Carrà, G., Johnson, S., 2009. Variations in rates of comorbid substance use in psychosis between geographical areas and mental health settings in the UK. Soc Psychiatry Psychiatr Epidemiol 44, 429-447. 
- Carrà, G., Johnson, S., Bebbington, P., Angermeyer, M.C., Heider, D., Brugha, T., Azorin, J.M., Toumi M., 2012. The lifetime and past-year prevalence of dual diagnosis in people with schizophrenia across Europe: findings from the European Schizophrenia Cohort (EuroSC). Eur Arch of Psychiatry Clin Neurosci 262, 607-616.

- Carrà, G., Scioli, R., Monti, M.C., Marinoni, A., 2006. Severity profiles of substance-abusing patients in Italian community addiction facilities: influence of psychiatric concurrent disorders. Eur Addict Res 12, 96-101.

- Carretta, D., Clerici, M., Bartoli, F., Carrà, G., 2015. Psychotic Disorders and Substance Use Disorders, in: el-Guebaly, N., Carrà, G., Galanter, M. (Eds.), Textbook of Addiction Treatment: International Perspectives. Springer, pp. 2013-2034.

- Dausch, B.M., Miklowitz, D.J., Richards, J.A., 1996. Global Assessment of Relational Functioning Scale (GARF): II. Reliability and validity in a sample of families of bipolar patients. Fam Process 35, 175-189.

- De Witte, N.A.J., Crunelle, C.L., Sabbe, B., Moggi, F., Dom G., 2014. Treatment for Outpatients with Comorbid Schizophrenia and Substance Use Disorders: A Review. Eur Addict Res 20, 105-114.

- Dervaux, A., Bayle, F.J., Krebs, M.O., 2002. Substance misuse among people with schizophrenia: similarities and differences between the UK and France. Br J Psychiatry 180, 381.

- Drake, R.E., Alterman, A.I., Rosenberg, S.R., 1993. Detection of substance use disorders in severely mentally ill patients. Community Ment Health J. 29, 175-192.

- Goldfinger, S.M., Schutt, R.K., Seidman, L.J., Turner, W.M., Penk, W.E., Tolomiczenko, G.S., 1996. Self-report and observer measures of substance abuse among homeless mentally ill persons in the cross-section and over time. J Nerv Ment Dis. 184, 667-672.

- Green, B., Young, R., Kavanagh, D., 2005. Cannabis use and misuse prevalence among people with psychosis. Br J Psychiatry 187, 306-313. 
- Hasin, D., Hatzenbuehler, M.L., Keyes, K., Ogburn, E., 2006. Substance use disorders: Diagnostic and Statistical Manual of Mental Disorders, fourth edition (DSM-IV) and International Classification of Diseases, tenth edition (ICD-10). Addiction 101 Suppl 1, 59-75.

- Hasin, D.S., O'Brien, C.P., Auriacombe, M., Borges, G., Bucholz, K., Budney, A., Compton, W.M., Crowley, T., Ling, W., Petry, N.M., Schuckit, M., Grant, B.F., 2013. DSM-5 criteria for substance use disorders: recommendations and rationale. Am J Psychiatry 170, 834-851.

- Heinrichs, D.W., Hanlon, T.E., Carpenter, W.T. Jr., 1984. The Quality of Life Scale: an instrument for rating the schizophrenic deficit syndrome. Schizophr Bull 10, 388-398.

- Jones, R.M., Lichtenstein, P., Grann, M., Långström, N., Fazel, S., 2011. Alcohol use disorders in schizophrenia: a national cohort study of 12,653 patients. J Clin Psychiatry 72, 775-779.

- Kerfoot, K.E., Rosenheck, R.A., Petrakis, I.L., Swartz, M.S., Keefe, R.S., McEvoy, J.P., Stroup, T.S.; CATIE Investigators, 2011. Substance use and schizophrenia: Adverse correlates in the CATIE study sample. Schizophr Res 132, 177-182.

- Kerner, B., 2015. Comorbid substance use disorders in schizophrenia: A latent class approach. Psychiatry Res 225, 395-401.

- Koskinen, J., Löhönen, J., Koponen, H., Isohanni, M., Miettunen, J., 2009. Prevalence of alcohol use disorders in schizophrenia--a systematic review and meta-analysis. Acta Psychiatr Scand 120, 85-96.

- Koskinen, J., Löhönen, J., Koponen, H., Isohanni, M., Miettunen, J., 2010. Rate of cannabis use disorders in clinical samples of patients with schizophrenia: a meta-analysis. Schizophr Bull 36, 1115-1130.

- Lehman, A.F., 1983. The effects of psychiatric symptoms on quality of life assessments among the chronic mentally ill. Eval Program Plann 6, 143-151.

- Lingford-Hughes, A.R., Welch, S., Peters, L., Nutt, D.J.; British Association for Psychopharmacology, Expert Reviewers Group., 2012. BAP updated guidelines: evidencebased guidelines for the pharmacological management of substance abuse, harmful use, addiction and comorbidity: recommendations from BAP. J Psychopharmacol 26, 899-952. 
- Margolese, H.C., Malchy, L., Negrete, J.C., Tempier, R., Gill, K., 2004. Drug and alcohol use among patients with schizophrenia and related psychoses: levels and consequences. Schizophr Res 67, 157-166.

- Mojtabai, R., 2005. Use of specialty substance abuse and mental health services in adults with substance use disorders in the community. Drug Alcohol Depend 78, 345-354.

- Morrens, M., Dewilde, B., Sabbe, B., Dom, G., De Cuyper, R., Moggi, F., 2011. Treatment outcomes of an integrated residential programme for patients with schizophrenia and substance use disorder. Eur Addict Res 17, 154-163. Norman, R.M.G., Malla, A.K., Cortese, L., Diaz, F., 1996. A study of the interrelationship between and comparative interraterreliability of the SAPS, SANS and PANSS. Schizophr Res 19, 73-85.

- Olfson, M., Lewis-Fernández, R., Weissman, M.M., Feder, A., Gameroff, M.J., Pilowsky, D., Fuentes M., 2002. Psychotic symptoms in an urban general medicine practice. Am J Psychiatry $159,1412-1419$.

- Potvin, S., Pampoulova, T., Mancini-Marië, A., Lipp, O., Bouchard, R., Stip, E., $2006 b$. Increased extrapyramidal symptoms in patients with schizophrenia and a comorbid substance use disorder. J Neurol Neurosurg Psychiatry 77, 796-798.

- Potvin, S., Sepehry, A.A., Stip, E., 2006a. A meta-analysis of negative symptoms in dual diagnosis schizophrenia. Psychol Med 36, 431-440.

- Rehm, J., Taylor, B., Room, R., 2006. Global burden of disease from alcohol, illicit drugs and tobacco. Drug Alcohol Rev 25, 503-513.

- Rosenberg, S.D., Goodman, L.A., Osher, F.C., Swartz, M.S., Essock, S.M., Butterfield, M.I., Constantine, N.T., Wolford, G.L., Salyers, M.P., 2001. Prevalence of HIV, hepatitis B and hepatitis C in people with severe mental illness. Am J Public Health 91, 31-37.

- Salyers, M.P., Mueser, K.T., 2001. Social functioning, psychopathology, and medication side effects in relation to substance use and abuse in schizophrenia. Schizophr Res 48, 109-123. 
- Schooler, N., Hogarty, G., Weissman, M., 1979. Social Adjustment Scale II (SAS-II), in: Hargreaves WA, Atkisson CC, and Sorenson JE. (Eds.), Resource materials for Community Mental Health Program Evaluations. NIMH, Rockville, MD, pp. 209-303.

- Simpson, S., Angus, R., 1970. A rating scale for extrapyramidal side effects. Acta Psychiatr Scand 212, 11-19.

- Soyka, M., Albus, M., Immler, B., Kathmann, N., Hippius, H., 2001. Psychopathology in dual diagnosis and non-addicted schizophrenics--are there differences? Eur Arch Psychiatry Clin Neurosci 251, 232-238.

- Spitzer, R.L., Williams, J.B., Gibbon, M., First, M.B., 1992. The Structured Clinical Interview for DSM-III-R (SCID). I: rationale, and description. Arch Gen Psychiatry 49, 624-629.

- Swartz, M.S., Wagner, H.R., Swanson, J.W., Stroup, T.S., McEvoy, J.P., Canive, J.M., Miller, D.D., Reimherr, F., McGee, M., Khan, A., Van Dorn, R., Rosenheck, R.A., Lieberman, J.A., 2006a. Substance use in persons with schizophrenia: baseline prevalence and correlates from the NIMH CATIE study. J Nerv Ment Dis 194, 164-172.

- Swartz, M.S., Wagner, H.R., Swanson, J.W., Stroup, T.S., McEvoy, J.P., McGee, M., Miller, D.D., Reimherr, F., Khan, A., Cañive, J.M., Lieberman, J.A., 2006b. Substance use and psychosocial functioning in schizophrenia among new enrollees in the NIMH CATIE study. Psychiatr Serv 57, 1110-1116.

- Talamo, A., Centorrino, F., Tondo, L., Dimitri, A., Hennen, J., Baldessarini, R.J., 2006. Comorbid substance-use in schizophrenia: Relation to positive and negative symptoms. Schizophr Res 86, 251-255.

- Tsai, J., Rosenheck, R.A., 2013. Psychiatric comorbidity among adults with schizophrenia: a latent class analysis. Psychiatry Res 210, 16-20.

- van Os, J., Bak, M., Hanssen, M., Bijl, R.V., de Graaf, R., Verdoux, H., 2002. Cannabis use and psychosis: a longitudinal population-based study. Am J Epidemiol 156, 319-327.

- Weaver, T., Madden, P., Charles, V., Stimson, G., Renton, A., Tyrer, P., Barnes, T., Bench, C., Middleton, H., Wright, N., Paterson, S., Shanahan, W., Seivewright, N., Ford, C.; Comorbidity 
of Substance Misuse and Mental Illness Collaborative study team., 2003. Comorbidity of substance misuse and mental illness in community mental health and substance misuse services. Br J Psychiatry 183, 304-313.

- Weiden, P., Rapkin, B., Mott, T., Zygmunt, A., Goldman, D., Horvitz-Lennon, M., Frances, A., 1994. Rating of medication influences (ROMI) scale in schizophrenia. Schizophr Bull 20, 297310.

- World Health Organization (WHO). SCAN: Schedules for Clinical Assessment in Neuropsychiatry. WHO Press, 1992. 